\title{
Clinical Spectrum of Hyponatremia in Patients with Stroke
}

\author{
Moiz Ehtesham ${ }^{1}$, Mawa Mohmand ${ }^{2}$, Kuldeep Raj ${ }^{3}$, Tooba Hussain ${ }^{4}$, FNU Kavita ${ }^{5}$, Besham Kumar ${ }^{6}$ \\ 1. Internal Medicine, Dow International Medical College, Dow University of Health Sciences, Karachi, PAK 2. Internal \\ Medicine, Khyber Medical College, Peshawar, PAK 3. Internal Medicine, Jinnah Sindh Medical University, Karachi, PAK \\ 4. Medicine, Dow University of Health Sciences, Karachi, PAK 5. Medicine, Liaquat University of Medical and Health \\ Sciences, Jamshoro, PAK 6. Internal Medicine, Jinnah Postgraduate Medical Center, Karachi, PAK
}

Corresponding author: Besham Kumar, beshamkumar916@gmail.com

\section{Abstract \\ Introduction}

Hyponatremia is a common electrolyte imbalance, which is readily observed in patients with ischemic as well as hemorrhagic stroke. It is mostly hypoosmolal and may be due to syndrome of inappropriate antidiuretic hormone (SIADH) or cerebral salt wasting syndrome (CSWS). The aim of this study was to evaluate the clinical spectrum of hyponatremia in patients of both hemorrhagic and ischemic strokes.

\section{Methods}

In this prospective observational study, all patients admitted with stroke were screened for serum sodium levels right after hospital admission. Patients with serum sodium levels $<135 \mathrm{mEq} / \mathrm{L}$ were included. Their demographic characteristics, type of stroke, etiology of hyponatremia, and site of hemorrhage/vascular territory ischemia was included.

\section{Results}

Hyponatremia was diagnosed in $34.2 \%$ of patients. Their mean serum sodium level was $130.4 \pm 3.5(\mathrm{mEq} / \mathrm{L})$. Ischemic stroke was more common in the hyponatremia group (67.7\%), and SIADH was a more common cause of hyponatremia (71.1\%). In hyponatremic patients with hemorrhagic stroke, right putamen hemorrhage was seen in $50 \%$ of patients with SIADH, and right thalamus was seen in $73.3 \%$ patients with CSWS. In hyponatremic patients with ischemic stroke, left middle cerebral artery ischemia was seen in $47 \%$ patients with SIADH and right middle cerebral artery ischemia was seen in 55\% patients with CSWS.

\section{Conclusion}

In patients with hyponatremia secondary to stroke, ischemic stroke is a common entity. SIADH remains a more frequently witnessed underlying pathology in hyponatremic stroke patients.

Received 07/24/2019

Review began 07/31/2019

Review ended 08/01/2019

Published 08/02/2019

\section{() Copyright 2019}

Ehtesham et al. This is an open access article distributed under the terms of the Creative Commons Attribution License CC-BY 3.0., which permits unrestricted use, distribution, and reproduction in any medium, provided the original author and source are credited.
Categories: Internal Medicine, Neurology, Miscellaneous

Keywords: stroke, hyponatremia, ischemic stroke, hemorrhagic stroke, severity, frequency

\section{Introduction}

Hyponatremia is a common electrolyte imbalance which is readily observed in patients of neurological disorders such as stroke. Hyponatremia is defined as serum sodium levels $\leqslant 135 \mathrm{mmol} / \mathrm{L}$ [1]. In stroke, hyponatremia is mostly hypoosmolal and may be caused by either syndrome of inappropriate anti-diuretic hormone (SIADH) or cerebral salt wasting syndrome (CSWS). SIADH is more common than CSWS [2]. It may also be due to dietary restriction of sodium as a measure to control hypertension, use of anti-hypertensive medicine such as diuretics, and secondary infections. It results in altered sensorium in these patients and may also predispose the patients to seizures.

In SIADH, there is an unchecked secretion of anti-diuretic hormone (ADH), from the posterior pituitary gland in response to stimulation from the hypothalamus. It causes body fluid hypotonicity and increased blood volume [2]. In CSWS, large quantities of sodium are lost in the urine [3].

Incidence of hyponatremia in stroke has been reported to be $11 \%$ to $35 \%$ in the literature $[2,4-5]$. The rate of mortality in hyponatremic stroke patients has been reported to be as high as 60\% [4]. In Pakistani literature, the frequency of hyponatremia has been reported to be as high as $35 \%$ to $45 \%$ in stroke patients; however, the mortality rate has been lower (16\%) as compared to other literature [6-7]. Mahesar et al. stated that only patients were ischemic stroke were included, and Shah et al. included only patients with hemorrhagic stroke [6-7]. In this study, we aimed to establish the frequency and severity of hyponatremia in patients of both hemorrhagic and ischemic strokes of all etiologies. 


\section{Cureus}

\section{Materials And Methods}

A prospective observational study was conducted in the Department of Neuromedicine of a tertiary care public hospital in Karachi. The study duration was 1st July to 31st December 2018. This study was approved by the institutional review board. Informed consent was taken from all patients.

During the study period, 354 patients, of both genders and age 18 years and above, were admitted with a clinical and neuro-radiological diagnosis of stroke. Strokes were classified as ischemic or hemorrhagic based on the findings of neuro-imaging by experienced neurologists. Hyponatremia was defined as serum sodium concentration $<135 \mathrm{mmol} / \mathrm{L}$ just after admission prior to administration of any medical therapy. The reason for this was that late-onset hyponatremia may be iatrogenic and not purely a consequence of the stroke itself. The underlying etiology was classified as SIADH or CSWS based on the classification described in Saleem et al. [2].

In patients with hemorrhagic stroke, the site of hemorrhage was identified on magnetic resonance imaging (MRI) or magnetic resonance angiography (MRA). The site of ischemia was determined on MRA. Where MRI or MRA was not possible or contraindicated, non-contrast computed tomography (NCCT) was done. SPSS for Windows version 22.0 (IBM Corp., Armonk, NY, US) was used to process the data.

\section{Results}

There were 226 (63.8\%) male and 128 (36.2\%) female patients. Their mean age was $28 \pm 11$ years (range: 24 68 years). There were 112 (31.6\%) patients of age 18-45 years. All patients were admitted from the emergency room. Hyponatremia was diagnosed in 121 (34.2\%) patients. The mean serum sodium level of these hyponatremic patients was $130.4 \pm 3.5(\mathrm{mEq} / \mathrm{L})$.

The characteristics of patients with hyponatremia are shown in Table 1. More patients were males and of older age group. Ischemic stroke was more common among hyponatremic patients and SIADH was a more common cause of hyponatremia, as seen in Table 1 .

Patient Characteristics

Gender

Male

Female

Age in years

18-45 years

$>45$ years

Known comorbidity status

Neurological disease

Hypertension

Ischemic heart disease

Diabetes mellitus

Type of stroke

Hemorrhagic stroke

Ischemic stroke

Cause of hyponatremia

SIADH

csws
39 (32.2\%)

$82(67.7 \%)$

$86(71.1 \%)$

Frequency (\%)

$73(60.3 \%)$

$48(39.7 \%)$

42 (34.7\%)

79 (65.3\%)

48 (39.6\%)

$28(23.1 \%)$

17 (14.1\%)

$9(7.4 \%)$

$35(28.9 \%)$

TABLE 1: Characteristics of stroke patients with hyponatremia ( $N=121)$

SIADH, syndrome of inappropriate secretion of antidiuretic hormone; CSWS, cerebral salt wasting syndrome 


\section{Cureus}

Among ischemic stroke patients with hyponatremia, SAIDH was established in 62 (75.6\%) patients and CSWS in 20 (24.4\%) patients. Comparatively, in hemorrhagic stroke patients with hyponatremia, SAIDH was established in 24 (61.5\%) patients and CSWS in 15 (38.5\%) patients. The mean serum sodium level in the ischemic group of hyponatremia was $128.2 \pm 1.8(\mathrm{mEq} / \mathrm{L})$ and that of the hemorrhagic group was $124.7 \pm 2.5$ $(\mathrm{mEq} / \mathrm{L})$.

The site of hemorrhage was then determined in patients with hemorrhagic stroke, as shown in Table 2. Right putamen was the most common site of hemorrhage in patients with SIADH and right thalamus was the most common in patients with CSWS. None of the patients in the SIADH group had pontine hemorrhage and only one in CSWS group had it (Table 2).

\begin{tabular}{|c|c|c|}
\hline Sites of hemorrhagic stroke & Hyponatremia due to SIADH $(n=24)$ & Hyponatremia due to CSWS $(n=15)$ \\
\hline Right putamen hemorrhage & $12(50 \%)$ & $7(46.7 \%)$ \\
\hline Left putamen hemorrhage & $9(37.5 \%)$ & $9(60 \%)$ \\
\hline Right thalamic hemorrhage & $3(12.5 \%)$ & $11(73.3 \%)$ \\
\hline Left thalamic hemorrhage & $8(33.3 \%)$ & $6(40 \%)$ \\
\hline Right cerebellar hemorrhage & $2(8.3 \%)$ & $3(20 \%)$ \\
\hline Left cerebellar hemorrhage & $1(4.2 \%)$ & --- \\
\hline Pontine hemorrhage & --- & $1(6.7 \%)$ \\
\hline
\end{tabular}

TABLE 2: Site of hemorrhage in hyponatremic patients with hemorrhagic stroke $(\mathrm{N}=39)$

SIADH, syndrome of inappropriate secretion of antidiuretic hormone; CSWS; cerebral salt wasting syndrome

Of all the patients of ischemic stroke with hyponatremia $(n=82)$, left middle cerebral artery (MCA) ischemia was the most common $(n=35 / 82 ; 42.6 \%)$, followed by right MCA ( $\mathrm{n}=33 / 82 ; 40.2 \%)$, and ischemia of anterior circulation was the least frequent $(n=14 / 82 ; 17.1 \%)$. The distribution of ischemic territory according to the type of hyponatremia is shown in Table 3.

Vascular Territory involved
Right Middle Cerebral Artery
Left Middle Cerebral Artery
Anterior Circulation

Hyponatremia due to SIADH $(n=62)$
$22(35.4 \%)$
$29(46.7 \%)$
$11(17.7 \%)$

Hyponatremia due to CSWS $(n=20)$
$11(55 \%)$
$6(30 \%)$
$3(15 \%)$

TABLE 3: Ischemic vascular territory in hyponatremic patients with ischemic stroke $(\mathrm{N}=\mathbf{8 2})$

SIADH, syndrome of inappropriate secretion of antidiuretic hormone; CSWS, cerebral salt wasting syndrome

Out of 121 hyponatremic patients, 81 (66.9\%) had mild hyponatremia (serum sodium: 130-134 mEq/L) and $62(76.5 \%)$ of them did not show any changes in mental and physical functioning. The remaining 29 (35.8\%) patients complained of generalized lethargy, malaise, excessive sleepiness, and nausea. Among the 31 (25.6\%) patients of moderate hyponatremia (serum sodium: 125-130 mEq/L), 22 (70.9\%) developed altered mental state, six (19.3\%) patients were lethargic with deep sleep (but not in coma), and three (9.7\%) patients developed pulmonary edema. Among the nine (7.4\%) patients with severe hyponatremia (serum sodium: $<125 \mathrm{mEq} / \mathrm{L}$ ), all patients developed an altered mental state, three (33.3\%) of them had an episode of generalized seizure, and two (22.2\%) patients developed cerebral edema.

\section{Discussion}

In this large-scale, single-center prospective study, the frequency of hyponatremia in stroke has been reported to be comparable to other data from the same region [6-7]. SIADH was reported as a more common entity. Ischemic strokes were more common than hemorrhagic ones. As with Shah et al., this study did not follow the patients for their hospital outcome and did not aim to establish any relationship between 
hyponatremia and mortality, which is a major limitation [7]. This study was time-bound with a limited number of patients.

Hyponatremia remains the most common electrolyte imbalance encountered in hospitalized patients. It is more frequently seen in patients with intracranial, both medical and surgical, disorders and severely ill patients admitted in the intensive care units. It may be due to the underlying disease; however, it may also be iatrogenic [8-9]. In most patients with neurologic disorders, hyponatremia is dilutional along with hypoosmolal serum, which induces cerebral edema. In hypertensive patients presenting with stroke, hyponatremia may also be due to pre-stroke diuretic therapy; however, no relationship has been established [9-10]. In an Iraqi study with $17 \%$ frequency of hyponatremia in stroke patients, all patients of hyponatremia suffered hemorrhagic stroke. Their clinical presentation included disturbed consciousness, headache, visual disturbances, and convulsions [11].

SIADH has been reported as a more common etiology in hyponatremia as compared to CSWS in the literature. In an Iraqi study with hyponatremic stroke patients, $82 \%$ had SIADH and $18 \%$ had CSWS [11]. In another study with a frequency of 35\% hyponatremia in patients with stroke, $67.5 \%$ had SIADH and $32.5 \%$ had CSWS. In hemorrhagic stroke group, $67 \%$ had SIADH and 33\% had CSWS. In the ischemic stroke group, 68.5\% had SIADH and 31.5\% had CSWS [2]. The results were comparable to our study. Pradhan et al. showed that mean sodium in the hemorrhagic group was lower than that in the ischemic group [12].

In this study, right putamen hemorrhage was more common in patients with SIADH and right thalamus hemorrhage was more common in patients with CSWS. None of the patients in the SIADH group had pontine hemorrhage. In hyponatremic ischemic stroke patients left MCA ischemia was the most common overall as well as in SIADH group. In the CSWS group, right MCA ischemia was more common. Saleem et al. also categorized their hyponatremic patients according to their site of hemorrhage and territory of vascular ischemia. Right MCA ischemia was the most common in CSWS group and left MCA was most commonly involved in the SIADH group. Left putamen hemorrhage was the most common in SIADH group and in CSWS group right putamen and right thalamus hemorrhage was equally seen. Overall, pontine hemorrhage was more common in their study as compared to ours [2]. Shah et al., in their patients with hemorrhagic stroke, reported right putamen hemorrhage to be more common in the SIADH group and left putamen in their CSWS group. The overall incidence of hyponatremia in their study was high - $45 \%$ - with $58.5 \%$ cases of SIADH and $41.5 \%$ of CSWS [7]. Mahesar et al. stated that the frequency of hyponatremia in patients with ischemic stroke was $38.6 \%$. Out of these cases, $64.7 \%$ had mild hyponatremia, $25.5 \%$ had moderate, and $9.8 \%$ had severe hyponatremia $[6]$.

Neurological disorders, including stroke, are frequently complicated by electrolyte imbalance. Serum sodium derangement remains the most commonly witnessed electrolyte imbalance in these patients. Hyponatremia is of prime clinical importance as its clinical presentation may mask the signs of already sustained neurological trauma in these patients. Early identification of rapidly declining serum sodium levels helps in initiating immediate measures in these patients.

\section{Conclusions}

Hyponatremia is a readily observed entity in patients with neurological disorders. In patients with hyponatremia secondary to stroke, ischemic stroke is a common entity. SIADH remains a more frequently witnessed underlying pathology in hyponatremic stroke patients. Severe hyponatremia may further deteriorate the neurological status of these patients. Early identification of rapidly declining serum sodium levels may help in immediate management.

\section{Additional Information}

\section{Disclosures}

Human subjects: Consent was obtained by all participants in this study. Dow University of Health Sciences issued approval ERC-014/2018. Animal subjects: All authors have confirmed that this study did not involve animal subjects or tissue. Conflicts of interest: In compliance with the ICMJE uniform disclosure form, all authors declare the following: Payment/services info: All authors have declared that no financial support was received from any organization for the submitted work. Financial relationships: All authors have declared that they have no financial relationships at present or within the previous three years with any organizations that might have an interest in the submitted work. Other relationships: All authors have declared that there are no other relationships or activities that could appear to have influenced the submitted work.

\section{References}

1. Adrogué HJ, Madias NE: The challenge of hyponatremia . J Am Soc Nephrol. 2012, 23:1140-1148. 10.1681/ASN.2012020128

2. Saleem S, Yousuf I, Gul A, Gupta S, Verma S: Hyponatremia in stroke. Ann Indian Acad Neurol. 2014, 17:5557. 10.4103/0972-2327.128554

3. Cerda'-Esteve M, Cuadrado-Godia E, Chillaron JJ, et al.: Cerebral salt wasting syndrome: review. Eur J Int 


\section{Cureus}

Med. 2008, 19:249-254. 10.1016/j.ejim.2007.06.019

4. Tisdall M, Crocker M, Watkiss J, Smith M: Disturbances of sodium in critically ill adult neurologic patients: a clinical review. J Neurosurg Anesthesiol. 2006, 18:57-63.

5. Huang WY, Weng WC, Peng TI, et al.: Association of hyponatremia in acute stroke stage with three-year mortality in patients with first-ever ischemic stroke. Cerebrovasc Dis. 2012, 34:55-62. 10.1159/000338906

6. Mahesar SA, Memon SF, Mustafa S, Javed A, Butt SM: Evaluation of hyponatremia in ischemic stroke patients in a tertiary care hospital of Karachi, Pakistan. Cureus. 2019, 11:e3926. 10.7759/cureus.3926

7. Shah A, Sabir S, Artani M, Salam O, Khan S, Rizwan A: Significance of hyponatremia as an independent factor in predicting short-term mortality in patients with hemorrhagic stroke. Cureus. 2019, 11:e4549. 10.7759/cureus. 4549

8. Coenraad MJ, Meinders AE, Taal JC, Bolk JH: Hyponatremia in intracranial disorders. Neth J Med. 2001, 58:123-127. 10.1016/S0300-2977(01)00087-0

9. Fofi L, Dall'Armi V, Durastanti L, et al.: An observational study on electrolyte disorders in the acute phase of ischemic stroke and their prognostic value. J Clin Neurosci. 2012, 19:513-516. 10.1016/j.jocn.2011.07.041

10. Joynt RJ, Feibel JH, Sladek CM: Antidiuretic hormone levels in stroke patients. Ann Neurol. 1981, 9:182-184. 10.1002/ana.410090212

11. Al-Khazraji AK: Hyponatremia in a group of Iraqi patients with stroke . Iraqi J Med Sci. 2016, 14:191-196.

12. Pradhan B, Majhi C, Panigrahi SK: Clinical profiles, electrolytes status in acute strokes and their outcome . Int J Adv Med. 2018, 5:492-497. 10.18203/2349-3933.ijam20181446 